\section{A single administration of 'microbial' D-alanine to healthy volunteers augments reaction to negative emotions: A comparison with D-serine}

\author{
Liliana P Capitão ${ }^{1,2}$, Jessica Forsyth ${ }^{1}$, Mia A Thomaidou1 ${ }^{10}$, \\ Mark D Condon ${ }^{1}$, Catherine J Harmer ${ }^{1,2}$ and Philip WJ Burnet ${ }^{1}$ (ID
}

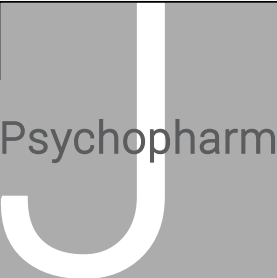

Journal of Psychopharmacology 2020, Vol. 34(5) 557-566 (c) The Author(s) 2020 Article reuse guidelines: sagepub.com/journals-permissions DOI: $10.1177 / 0269881120908904$ journals.sagepub.com/home/jop

@SAGE

\begin{abstract}
Background: Activation of the glutamate N-methyl-D-aspartate receptor with its co-agonist D-serine has been shown to improve subjective mood in healthy volunteers. D-alanine is another potent $\mathrm{N}$-methyl-D-aspartate receptor co-agonist which arises from the natural breakdown of host gut microbes, and is predominantly sequestered in the pituitary. This may suggest that D-alanine influences the neuroendocrine stress response which may then impact on emotion.

Aims: The current study explored the effects of D-serine and D-alanine on emotional processing, cognition and the levels of the stress hormone cortisol in healthy volunteers.

Methods: In a double-blind, placebo-controlled randomised study, participants ( $n=63$ ) received a single oral dose of either D-serine, D-alanine $(60 \mathrm{mg} / \mathrm{kg})$ or placebo and then performed the Emotional Test Battery and N-back task (two hours post-administration) and provided saliva samples at fixed intervals.

Results: Subjects administered with D-alanine were faster at identifying facial expressions of fear, surprise and anger, and at categorising negative self-referential words. Participants on D-alanine also showed a trend to recall more words than placebo in a memory task. D-serine did not have any meaningful effects in any of the tasks. Neither amino acid had a significant effect on salivary cortisol or working memory.

Conclusion: This study is the first to suggest that D-alanine can modulate emotional cognitive processing after a single dose. The lack of findings for D-serine nevertheless contrasts a previous study, emphasising a need for further investigation to clarify discrepancies. A better understanding of the physiological actions of D-amino acids would be beneficial in evaluating their therapeutic potential.
\end{abstract}

\title{
Keywords
}

D-amino acids, cognition, emotional processing, cortisol

\section{Introduction}

The glutamate N-methyl-D-aspartate receptor (NMDAR) is crucial for forming and maintaining synaptic plasticity in neural regions involved in cognition, memory and emotional processing (Grunze et al., 1996; Lohmann and Kessels, 2014; Meuwese et al., 2013). Some negative symptoms seen in schizophrenia, such as flattened affect and cognitive deficits, have been associated with compromised NMDAR signalling (Coyle, 2012; Javitt, 2009; Rubio et al., 2012) and activation of this receptor with its co-agonist, D-serine, has shown therapeutic potential (Guercio and Panizzutti, 2018; Hashimoto et al., 2013). Additionally, there is emerging evidence that NMDAR may be a potential target for mood disorders (Sanacora et al., 2008) and, intriguingly, both antagonism (with ketamine) and stimulation (with D-serine) of the NMDAR appear to have anxiolytic/antidepressant effects (Berman et al., 2000; Chan et al., 2016).

D-serine is a full agonist of the glycine site of the NMDA receptor (MacKay et al., 2019), being present endogenously in several cortico-limbic regions (Wolosker et al., 2008). The effects of D-serine have been explored in both animal and human samples. In rodents, a single administration of D-serine has been shown to promote antidepressant-like behaviour in chronically stressed mice (Malkesman et al., 2012). In healthy volunteers, a single oral dose of D-serine not only reduced subjective feelings of sadness and anxiety, but also improved attention and vigilance as well as verbal memory (Levin et al., 2015). Consistent with these findings, clinical studies have shown that D-serine administration improves both negative symptoms and neuropsychological functioning in patients with schizophrenia (Kantrowitz et al., 2010). These observations support the notion of NMDAR co-agonist activation as a potential therapeutic strategy for mood and cognitive disorders.

The D-amino acid D-alanine is also a NMDAR endogenous coagonist, albeit present at lower levels in the brain, and has been shown to improve both negative symptoms (flattened affect, social

${ }^{1}$ Department of Psychiatry, University of Oxford, Oxford, UK ${ }^{2} 0 x f o r d$ Health NHS Foundation Trust, 0xford, UK

\section{Corresponding author:}

Philip WJ Burnet, Department of Psychiatry, University of 0xford, Neuroscience Building, Warneford Hospital, Oxford, 0X3 7JX, UK. Email: phil.burnet@psych.ox.ac.uk 
withdrawal) and cognition in patients with schizophrenia, following repeated treatment (Tsai et al., 2006). Immunohistochemistry has shown concentrations of D-alanine in the anterior pituitary and pineal gland, endocrine structures that hormonally regulate stress and sleep-wake cycles (Hamase et al., 2005). This is of interest considering the association between stress and cortisol with disorders such as depression, anxiety and schizophrenia (Girshkin et al., 2014; Herbert, 2013). Acute doses of cortisol in vitro disrupt NMDAR function and impair synaptic plasticity, which may underlie the detrimental effects of chronic stress on mood and learning (Bennett A $\mathrm{O}, 2008)$. If $\mathrm{D}$-alanine reduces cortisol in humans, it could provide an additional therapy for stress-related disorders.

There is, therefore, sufficient evidence to warrant further investigation into the psychological actions of both $\mathrm{D}$-serine and D-alanine in humans. One approach is to investigate changes in emotional and cognitive processing using a battery of well-validated tasks, following a single administration of the D-amino acid. Drugs that are widely used to treat psychiatric disorders can produce changes in emotional and cognitive processes at a single dose. For instance, a single dose of the antidepressant drugs citalopram or mirtazapine produces a robust positive emotional bias in tasks measuring facial expression recognition and/or memory in healthy volunteers (Arnone et al., 2009; Browning et al., 2007; Harmer et al., 2003). Similarly, the cognitive-enhancing drug modafinil has been shown to increase implicit learning after a single dose (Winder-Rhodes et al., 2010). These findings suggest that standard pharmacotherapies act early to modulate the psychological paths that underlie the symptoms of psychiatric disorders, before any measurable effects on symptoms are seen. Therefore, this psychological approach provides a method to investigate potential effects of novel drugs.

In the current study, participants were therefore administered a battery of well-standardised psychological tasks aimed at assessing emotional processing and cognition (Capitão et al., 2015; Harmer et al., 2011), following a single dose of D-serine, D-alanine or placebo $(60 \mathrm{mg} / \mathrm{kg})$. The study had four objectives. First, to test if a single administration of D-serine would improve cognition (specifically attention, vigilance and memory) as suggested by Levin et al. (2015). Second, to test if D-serine would also increase processing of positive vs negative information, consistent with a potential antidepressant-like action, given preliminary evidence suggesting that D-serine may improve mood and negative symptoms. Third, to examine the psychological effects of a single dose of D-alanine for the first time. Fourth, to explore the effects of D-alanine and D-serine on salivary cortisol levels during psychometric testing.

\section{Materials and methods}

\section{Participants}

Healthy participants ( $n=63,30$ males, 33 females, aged 18-55 years) were randomly allocated to placebo $(n=21$, nine male), D-alanine ( $n=22,11$ male) or D-serine groups $(n=20,10$ male). The sample size was determined based on a previous study which found that a single dose of an antidepressant reduced accuracy to detect fearful faces, with an effect size of 1.09 (Harmer et al., 2004). Based on this study, it was determined that the minimum sample size required to detect changes in accuracy (difference between two independent means: two-tailed, alpha $=0.05$, effect size $=1.09$, power $=0.9$ ) was $n=19$ per group.
Participants were recruited by local advertisement. Exclusion criteria included: a history of psychological disorder (i.e. depression, anxiety), substance misuse or use of psychotropic medication in the last three months, dyslexia, prior exposure to task battery, current pregnancy or breastfeeding, gastro-intestinal problems, or a body mass index (BMI) outside the range of 18 $30 \mathrm{~kg} / \mathrm{m}^{2}$. All interested participants were screened using the Structural Clinical Interview for the DSM-IV Axis I Disorders (SCID-I; First et al, 1996) to exclude for current/past psychiatric disorders. Recruitment was balanced for gender.

\section{Experimental design}

The investigation was run as a double-blind, randomised, parallel and placebo-controlled study, which received ethical approval by the University of Oxford Central University Research Ethics Committee (reference MSD-IDREC-C3-2014-05).

Testing of all participants started between 09:00-10:00. Participants were asked to have fat-free breakfasts and refrain from alcohol, caffeine and major exercise $24 \mathrm{~h}$ prior to testing. After providing a baseline saliva sample and completing selfreport questionnaires, participants received a single dose of D-alanine $(60 \mathrm{mg} / \mathrm{kg})$, D-serine $(60 \mathrm{mg} / \mathrm{kg}$ ) or a placebo (Stevia, artificial sweetener) dissolved in $100 \mathrm{~mL}$ of water. The choice of dose was based on a previous study with patients with schizophrenia where $60 \mathrm{mg} / \mathrm{kg}$ was the lowest concentration of D-serine that initiated a significant change in negative symptoms (Harmer et al., 2004). Parenthetically, the Levin et al. study (2015) used an acute dose of $2.1 \mathrm{~g}$ fixed for all participants - our doses ranged from $2.7-4.9 \mathrm{~g}$ for participants weighing $45-80 \mathrm{~kg}$ respectively. D-serine has been shown to peak in serum after two hours (Kantrowitz et al., 2010; Levin et al., 2015), and so computerised battery tests were performed two hours post-administration; these included standardised emotional processing and cognitive tasks. Self-report questionnaires were completed alongside saliva samples at baseline before the drink, and at one, two and four hours post-drink.

\section{Subjective symptoms}

On the testing day, baseline subjective symptoms were selfassessed using the Beck Depression Inventory (BDI), State-Trait Anxiety Inventory (STAI), Profile of Mood States (POMS), Positive and Negative Affective Schedule (PANAS) and Befindlichkeits Scale (BFS). The STAI-State, PANAS, BFS and POMS were repeated one, two and four hours post-drink to track potential changes in subjective feelings. At the end of the study, participants reported how well they were feeling using a singleitem visual analogue scale and were also asked to guess which treatment they had received (D-amino acid or placebo).

\section{Psychological tasks}

The Emotional Test Battery (ETB) comprises five tasks measuring a participant's bias towards positive or negative valence facial expressions and words. It has been used previously to investigate the cognitive and emotional processing effects of psychopharmacological agents (Harmer et al., 2011). Participants were administered the ETB tasks in this order: Facial Expression 
Table 1. Treatment group characteristics.

\begin{tabular}{|c|c|c|c|c|}
\hline Demographic & Placebo $(n=21)$ & D-alanine $(n=22)$ & D-serine $(n=20)$ & $p$ \\
\hline Age; mean (SD) & $27.14(6.73)$ & $26.50(7.96)$ & $28.60(9.03)$ & 0.747 \\
\hline Gender; $n$ female $(\%)$ & $12(57 \%)$ & $11(50 \%)$ & $10(50 \%)$ & 0.286 \\
\hline BMI; mean (SD) & $22.54(2.54)$ & $22.40(2.15)$ & $23.48(2.75)$ & 0.518 \\
\hline Education level; mean (SD) & $2.24(0.83)$ & $2.32(0.95)$ & $2.40(0.88)$ & 0.103 \\
\hline Native speaker; $n$ native (\%) & $14(67 \%)$ & $16(87 \%)$ & $14(70 \%)$ & 0.283 \\
\hline
\end{tabular}

ANOVA: analysis of variance; BMI: body mass index; NART: National Adult Reading Test; SD: standard deviation.

Education level: ' 1 ' A-levels, ' 2 ' Undergraduate, ' 3 ' Masters, ' 4 ' PhD. Values of $p$ were assessed by one-way ANOVA or $\chi^{2}$-test as appropriate.

Recognition Task (FERT) (lasting approximately $15 \mathrm{~min}$ ), Emotional Categorisation Task (ECAT) (5 min), Attentional DotProbe (15 min), Emotional Recall (EREC) (2 min) and Emotional Recognition (EMEM) (15 min). The N-back task (assessing working memory, lasting $12 \mathrm{~min}$ ) was the final task to be administered. Altogether, the completion of these tasks lasted approximately 70 minutes. Each task is summarised for clarity preceding the appropriate results section, with detailed methods in the Supplementary Material.

\section{Salivary cortisol}

Participants provided four saliva samples, at baseline and one, two and four hours after the administration of D-amino acid or placebo. Saliva was frozen post-testing. To assay, samples were thawed and centrifuged (1500 $\mathrm{g}$ for $15 \mathrm{~min}$ ) and assessed using a commercial cortisol enzyme-linked immunosorbent assay (ELISA) kit according to manufacturer's instructions (Salimetrics, UK).

\section{Statistical analysis}

Data was analysed using SPSS v21. Baseline characteristics were analysed using one-way analyses of variance (ANOVAs) or $\chi^{2}$ tests where appropriate. All other measures were analysed by mixed-design ANOVAs, where group (D-alanine, D-serine and placebo) was the between-subject factor. For subjective states, time and scale (where appropriate) were the within-subject factors. For the FERT, ECAT and EREC, emotion was the within-subject factor. For the Dot-Probe, within-subject factors were valence and masking. For the N-Back, the within-subject factor was difficulty rating (0-3). Where sphericity was violated, Greenhouse-Geisser corrected $p$ values were used. Significant interactions were explored using Bonferroni-corrected pairwise comparisons, adjusted for the two main comparisons of interest (D-alanine vs placebo and D-serine vs placebo). A value of $p<0.05$ denotes statistical significance. Marginal differences with a $p$-value lower than 0.10 were also reported. Effect size was estimated using $\eta p^{2}$.

\section{Results}

\section{Participants}

There was no significant difference between groups in terms of age, gender, BMI, education level or number of native speakers $(p>0.3)$ (Table 1). On the testing day, baseline mood did not significantly differ between groups, as assessed by one-way ANOVAs of STAI (trait and state), BDI, PANAS, BFS and POMS ( $p>0.3)$ (Table 2).

\section{FERT}

In the FERT, participants categorised images of human faces expressing seven emotions (happy, angry, surprise, sad, fear, disgust and neutral) and were assessed for accuracy, number of misclassifications and reaction times (RTs). Percentage misclassification were calculated as the total number of misclassifications per participant divided by the total misclassifications in each emotion category. A placebo participant (male) was excluded in the RT analysis for showing RTs three standard deviations (SDs) away from the group mean.

Accuracy and misclassifications. There was no significant interaction with treatment group and emotion $\left(F_{(12,360)}=0.977\right.$, $p=0.470)$ or main effect of group $\left(F_{(2,60)}=0.473, p=0.625\right)$ on accuracy (Figure 1(a)). A significant main effect of emotion was found $\left(F_{(6,360)}=37.793, p<0.001, \eta p^{2}=0.39\right)$, with neutral and happy faces most easily identified, and anger, fear and sadness the least (Figure 1(a)). There was no main effect of group or interaction with emotion on faces commonly falsely recognised $(p>0.8)$.

RTs. There was a significant interaction between emotion and group for RTs $\left(F_{(12,348)}=2.183, p=0.023, \eta p^{2}=0.070\right)$. Follow-up pairwise comparisons revealed the $\mathrm{D}$-alanine group as significantly faster than placebo at detecting anger $(p=0.044)$, fear $(p=0.024)$ and surprise $(p=0.028)$ (Figure 1(b)).

\section{ECAT}

In the ECAT, participants classified a series of self-referential personality adjectives as being either 'likeable' (i.e. friendly, thoughtful) or 'dislikeable' (i.e. dull, manic), and were measured for accuracy in categorisation and RTs to respond. RTs were the main outcome measure in this task.

ECAT accuracy. A significant main effect of group was found for accuracy of classification in the $\operatorname{ECAT}\left(F_{(2,59)}=5.649\right.$, $p=0.006, \eta p^{2}=0.161$ ) (Figure 2(a)). Follow-up pairwise comparisons showed the D-alanine group as significantly more accurate than the placebo group at categorising words ( $p=0.004 ; 96.43 \pm 0.9$ vs $92.38 \pm 0.9$, respectively). The $\mathrm{D}$-serine group was also more accurate than the placebo group 
Table 2. Subjective symptoms scores of participants on testing day.

\begin{tabular}{lccc}
\hline Questionnaire & Placebo $(n=21)$ & D-alanine $(n=22)$ & D-serine $(n=20)$ \\
\hline STAI-trait; baseline & $29.17(5.46)$ & $29.94(6.34)$ & $31.30(8.85)$ \\
BDI; baseline & $1.84(2.22)$ & $1.83(2.28)$ & $2.40(3.22)$ \\
STAI-state; baseline & $26.13(4.41)$ & $26.82(5.32)$ & $28.11(5.84)$ \\
One hour & $26.38(3.34)$ & $26.06(5.70)$ & $26.74(7.42)$ \\
Two hours & $25.38(3.74)$ & $27.06(6.08)$ & $26.68(6.68)$ \\
Four hours & $27.63(5.56)$ & $27.76(7.38)$ & $29.80(7.17)$ \\
PANAS-positive; baseline & $32.11(6.01)$ & $29.65(8.59)$ & $31.89(8.57)$ \\
One hour & $29.89(7.59)$ & $29.35(7.58)$ & $30.37(7.16)$ \\
Two hours & $30.89(7.08)$ & $29.12(8.46)$ & $29.63(9.69)$ \\
Four hours & $29.63(6.63)$ & $30.53(8.68)$ & $28.37(8.29)$ \\
PANAS-negative; baseline & $11.79(2.97)$ & $10.76(1.72)$ & $11.32(1.92)$ \\
One hour & $13.58(6.20)$ & $10.64(1.46)$ & $10.84(1.83)$ \\
Two hours & $11.00(1.49)$ & $10.47(1.28)$ & $10.47(1.50)$ \\
Four hours & $10.74(2.08)$ & $10.41(1.06)$ & $11.53(3.06)$ \\
BFS-total: baseline & $11.00(8.54)$ & $13.76(15.18)$ & $13.25(11.84)$ \\
One hour & $14.63(14.24)$ & $12.82(13.13)$ & $13.40(12.04)$ \\
Two hours & $10.68(10.08)$ & $11.41(8.95)$ & $12.35(11.80)$ \\
Four hours & $12.58(10.20)$ & $8.94(8.52)$ & $19.25(18.22)$ \\
POMS-TMD baseline & $-11.65(14.07)$ & $-4.53(10.58)$ & $-5.95(11.58)$ \\
One hour & $-9.18(15.83)$ & $-7.24(9.85)$ & $-8.42(10.69)$ \\
Two hours & $-12.24(12.58)$ & $-9.06(9.90)$ & $-7.53(12.87)$ \\
Four hours & $-10.24(14.98)$ & $-8.65(12.21)$ & $-3.26(12.25)$ \\
\hline
\end{tabular}

ANOVA: analysis of variance; BDI: Beck Depression Inventory; BFS: Befindlichkeits Scale (assessing mood); PANAS: Positive and Negative Affective Schedule; POMS-TMD: Profile of Mood States-Total Mood Disturbance; STAI: State-Trait Anxiety Inventory.

Values of $p$ were assessed using ANOVAs with time and scale (where appropriate) as within-subject factors and group as between-subject factor - no significant main effects or interactions were found.

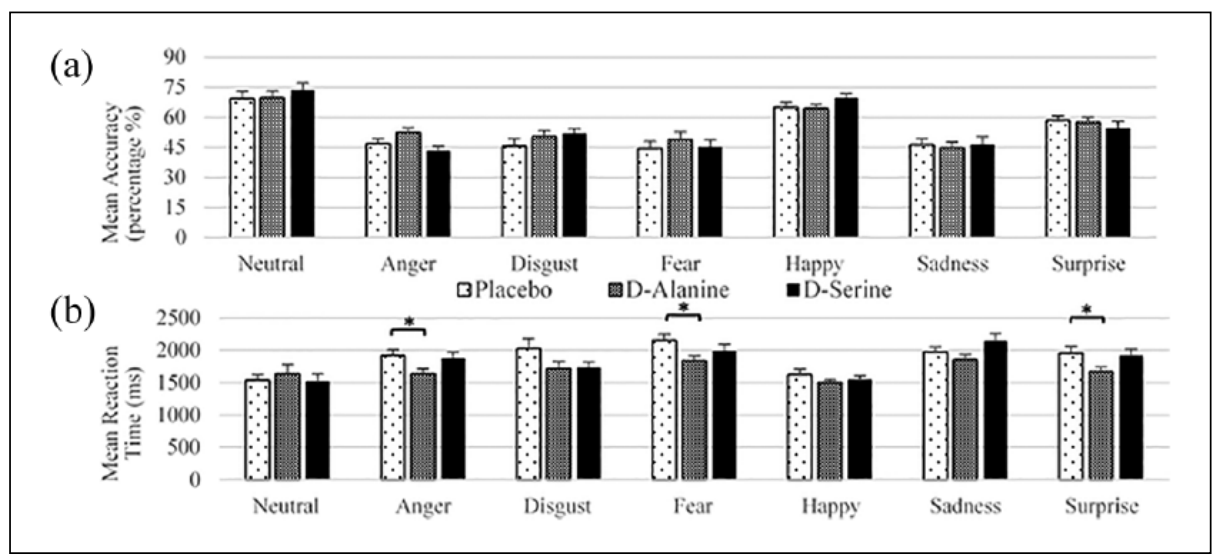

Figure 1. Facial Expression Recognition Task (FERT) scores following D-amino acid treatment. (a) Accuracy: no significant differences between groups. (b) Reaction time: D-alanine group was faster than placebo at identifying anger, fear and surprise. Error bars refer to standard error of the mean. ${ }^{*} p<0.05$.

( $p=0.04 ; 95.42 \pm 1.0$ vs $92.38 \pm 0.9$ ) (Figure $2(a)$ ). There was no significant interaction between group and valence of the word $\left(F_{(2,59)}=0.183, p=0.833\right)$. There was a significant main effect of valence, however $\left(F_{(1,59)}=4.880, p=0.031, \eta p^{2}=0.076\right)$, with a greater accuracy overall for categorising positive words than negative words $(p=0.031)$ (data not shown).

ECAT RT. RTs were the main outcome measure in this task. There was a significant interaction between treatment group and valence of word for RTs $\left(F_{(2,59)}=5.783, p=0.005, \eta p^{2}=0.164\right)$. Follow-up pairwise comparisons showed the D-alanine group were significantly faster than placebo at categorising words of negative valence $(p=0.012)$ (Figure $2(b))$. No significant differences were seen for positive words $(p=0.204)$ or for D-serine $(p>0.2)$. There was also a trend for a main effect of group on RT $\left[\mathrm{F}(2,59)=3.003, p=.057, \eta \mathrm{p}^{2}=.092\right]$, with D-alanine showing faster RTs overall than placebo $(p=0.038)$ (Figure 2(c)). Finally, there was a main effect of emotion $\left(F_{(1,59)}=41.187, p<0.001\right.$, 


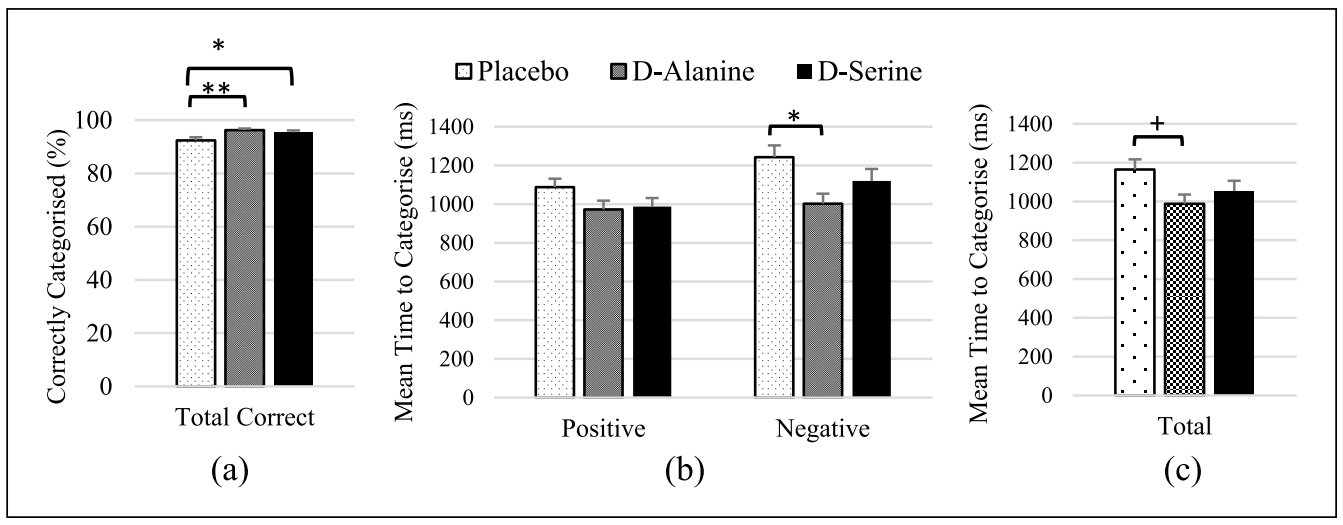

Figure 2. Emotional Categorisation Task (ECAT) scores following D-amino acid administration. (a) Accuracy: both D-alanine and D-serine groups were significantly more accurate at categorising words compared to placebo. (b) Reaction time: D-alanine was faster than placebo for words of negative valence. (c) D-alanine was also trending faster than placebo to categorise words regardless of valence. Error bars refer to standard error of the mean.

${ }^{* *} p<0.01 ;{ }^{*} p<0.05 ;+p<0.10$.

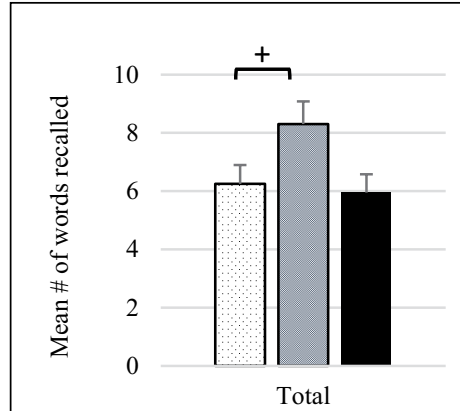

(a)

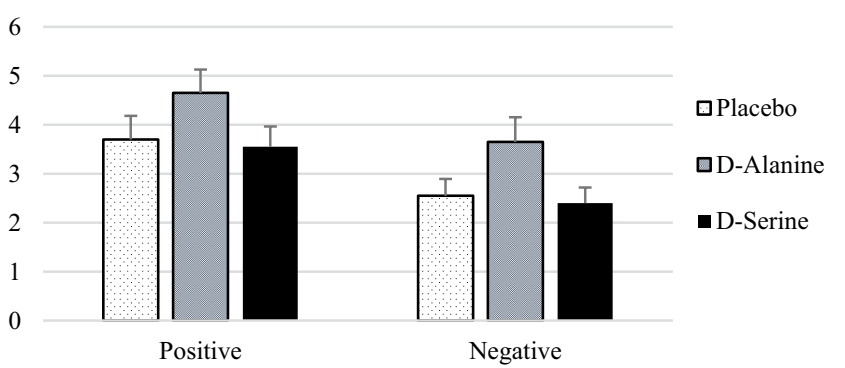

(b)

Figure 3. Results from Emotional Recall (EREC) (Free Recall) task for words of positive and negative valence after administration with D-amino acid. (a) D-alanine group showed a trend for a greater number of words recalled than placebo. (b) No significant interaction between group and emotion was found. Error bars refer to standard error of the mean. $+p<0.10$.

$\left.\eta \mathrm{p}^{2}=0.411\right)$, with positive words eliciting a faster RT than negative words $(p<0.001)$ (data not shown).

\section{EREC and EMEM}

The EREC and EMEM assess short-term memory of words from the ECAT; the EREC is a two-minute free-recall task (measured for accuracy), while the EMEM is computerbased, and requires participants to distinguish familiar ECAT words from novel distractor words (measured for accuracy and RTs).

EREC. There was a main effect of group for number of words correctly free-recalled $\left(F_{(2,57)}=3.460, p=0.038, \eta p^{2}=0.108\right)$, however follow-up pairwise comparisons revealed only a trend for D-alanine to recall more words than placebo $(p=0.078)$ (Figure 3(a)). No significant differences were seen between D-serine and placebo $(p>1.0)$. There was no significant interaction of group with valence of word $\left(F_{(2,57)}=0.028, p=0.972\right)$. There was no main effect of group or interaction between valence and group when considering the number of intrusions $(p>0.55)$. Overall, a main effect of valence was found $\left(F_{(1,57)}=13.744, p<0.001\right.$, $\eta \mathrm{p}^{2}=0.194$ ), with positive words recalled more than negative ones (Figure 3(b)).

EMEM. There was no significant main effect of group on accurately remembering words $\left(F_{(2,60)}=0.431, p=0.652\right)$, nor a significant interaction between group and emotion $\left(F_{(2,60)}=2.000\right.$, $p=0.144)$. There was also no main effect of group on RT $\left(F_{(2,60)}=0.163, p=0.850\right)$ or interaction between group and emotion $\left(F_{(2,60)}=2.286, p=0.110\right)$. There was a significant main effect of emotion however $\left(F_{(1,60)}=111.591, p<0.001, \eta \mathrm{p}^{2}=0.650\right)$, with positive words eliciting a faster RT than negative words $(p<0.001)$ (data not shown).

\section{Attentional Dot-Probe task}

The Dot-Probe task measures attentional vigilance to positive (happy) and negative (fearful) faces vs neutral. There was no significant main effect of group, nor any interaction with valence or masking (all $p>0.3$ ). The groups also did not differ in their overall accuracy $\left(F_{(2,59)}=1.105, p=0.338\right)$. 


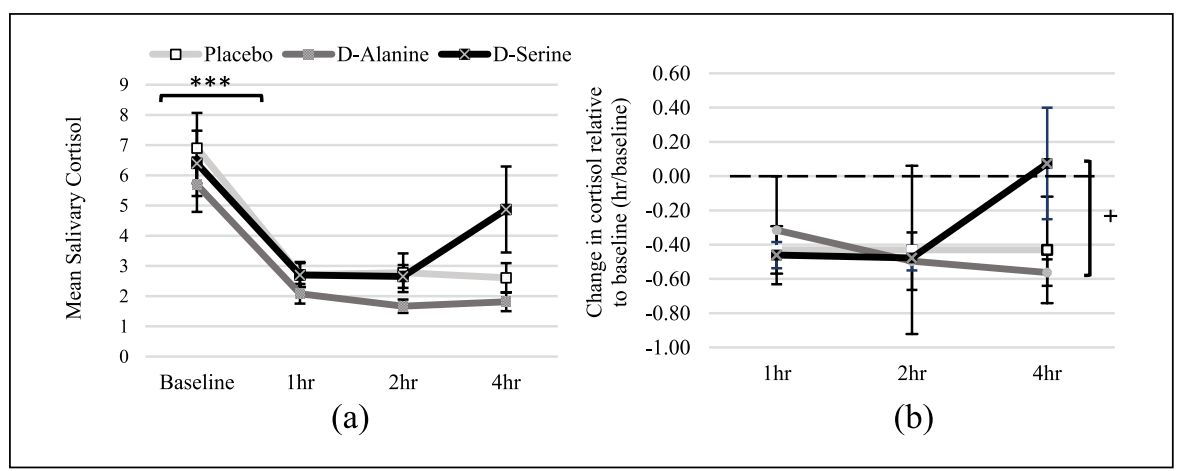

Figure 4. Salivary cortisol following D-amino acid ingestion. (a) Cortisol significantly decreased one-hour post-treatment in all groups. (b) No significant interactions were observed when individual cortisol concentrations were expressed as a ratio of respective baselines ((cortisol at hour $x$ )/ baseline). Dotted line represents baseline. Error bars refer to standard error of the mean.

${ }^{* * *} p<0.01,+p<0.10$.

\section{N-Back task}

The N-Back task assesses working memory; participants are presented with a sequence of letters and must compare a current letter to one previously in the series, with three levels of difficulty. Accuracy at these three difficulty levels were expressed as relative ratios to the control condition.

No significant main effect of treatment group was found for accuracy or RT $(p>0.3)$. Overall, there was a main effect of difficulty $\left(F_{(2,98)}=50.508, p<0.001\right)$ (data not shown), with comparisons at the first stage of difficulty significantly more accurate than stages 2 or $3(p<0.001)$, but with no differences between the second and the latter $(p=0.648)$.

\section{Salivary cortisol}

Salivary cortisol was assayed in samples collected at baseline and one, two and four hours post-treatment. One D-alanine participant was excluded for a baseline cortisol analysis because they were over four SDs from the group mean. One-way ANOVA found no significant difference in baseline cortisol levels between groups $\left(F_{(2,60)}=0.332, p=0.719\right)$.

A mixed-effects ANOVA with time as within-subject factor showed no significant main effect of treatment group on cortisol levels $(p>0.1)$, or interaction over time $(p>0.3)$ (Figure 4(a)). To correct for individual variation, cortisol at hours 1,2 and 4 were expressed as relative ratios to baseline (i.e. hour $x$ /baseline), and assessed again. There was still no significant effect of group on cortisol or interaction over time (Figure 4(b)) $(p>0.05)$. Overall, there was a main effect of time $\left(F_{(3,174)}=21.822, p<0.001, \eta p^{2}=0.273\right)$, with cortisol levels for all groups decreasing significantly one hour post-treatment $(p<0.001)$ (Figure 4(a)). Because of the high baseline cortisol levels in all groups, follow-up one-way ANOVAs were performed comparing means for hours 1,2 and 4 . Cortisol at hour 4 showed a significant main effect of group $\left(F_{(2,60)}=3.236, p<0.047\right)$ with D-serine trending for higher cortisol compared to D-alanine ( $p=0.052)$ (Figure 4(b)), but this did not extend when compared to placebo $(p=0.216)$.

\section{Subjective symptoms}

There was no significant main effect of treatment group on scores for the STAI-State, PANAS (positive and negative), BFS (for energy, mood or total) or POMS (in any sub-dimension or total), nor any significant interaction of treatment with time $(p>0.05)$ (see Table 2). One-way ANOVA showed the D-serine group reporting feeling less well than placebo on the 'wellness' analogue scale post-testing $(p<0.05)$.

When participants were asked to choose which group they believed they were in, their choices were independent of their actual groups $(p>0.4)$. This suggests that blinding was effective in this study.

\section{Discussion}

This study explored the effects of a single dose of D-serine and D-alanine on emotional processing, cognition and salivary cortisol levels in healthy volunteers. D-alanine increased speed to respond to negative emotions, which suggested an apparent negative bias. There was also a trend for D-alanine to increase the free recall of emotional words. Contrary to a previous report (Levin et al., 2015), D-serine had no significant effects on cognition. There were no significant effects of either D-amino acid on salivary cortisol levels. The current investigation shows, for the first time, an effect of D-alanine that is distinct from D-serine. The interpretation of these findings is discussed below.

\section{The effects of D-alanine on emotional processing and cognition}

Our current findings suggest that D-alanine administration may facilitate the processing of emotionally-charged information. D-alanine increased the accuracy to correctly classify words and showed a trend to improve their subsequent free-recall. D-alanine was also shown to reduce the speed to respond to facial expressions (in the FERT) and self-referential words (in the ECAT).

It is interesting to note that the effects of D-alanine, whilst subtle, were significant for stimuli with a negative valence. In the FERT, participants on D-alanine were faster to detect fear and anger, which convey negative emotions; surprise is sometimes perceived as negative (Noordewier and Breugelmans, 2013). Similarly, in the ECAT, the D-alanine group was significantly faster at categorising 'dislikeable' words, with no difference for 
'likeable' words. These observations can be interpreted in several ways.

One possibility is that a single dose of D-alanine induces a negative bias by increasing salience of negative information, which may suggest a potential depressogenic and/or anxiogenic action. This stands in contrast with our a priori hypothesis, as we predicted an increase in positive bias; however, it is interesting to note that some drugs widely used as antidepressants have been shown to paradoxically increase the processing of negative information after an acute dose, a phenomenon that is reversed with longer treatment courses. For instance, the commonly prescribed antidepressant drug citalopram has been reported to increase accuracy to detect fearful stimuli following a single administration (Browning et al., 2007), and this is thought to reflect the increase in anxiety frequently seen clinically when patients initiate antidepressant treatment. It is possible that D-alanine facilitates the processing of negative information following a single dose, but the interpretation of this finding is difficult as the clinical effects of D-alanine in humans have only been explored after repeated administration.

Alternatively, this finding could reflect a subtle pro-cognitive effect in the performance of these tasks, consistent with evidence suggesting that D-alanine may have pro-cognitive effects in patients with schizophrenia (e.g. Tsai et al., 1998), which may interact more strongly with negative emotions. Indeed, positive emotions are easier to process and reach a ceiling level more easily. There is some support for this in the current data. In the FERT, positive emotions (happy faces) had the highest accuracy rate and fastest RTs regardless of treatment (Figure 1), and similarly in the ECAT, positive words elicited greater accuracy and faster RTs overall (Figure 2). It is therefore possible that a depressogenic/anxiogenic property of D-alanine may co-exist with a subtle pro-cognitive action.

Mice with high levels of D-alanine and D-serine following genetic deletion of the $\mathrm{D}$-amino acid metabolic enzyme (D-amino acid oxidase (DAO)) show enhanced cognitive performance in memory tests, but also increased anxiety-like behaviours (Labrie et al., 2009a, 2009b; Miyoshi et al., 2011). Therefore, both effects could co-exist such that a single dose of D-alanine might lead to both pro-cognitive and depressogenic/anxiogenic effects (Chan et al., 2016). However, a depressogenic effect contradicts findings that chronic doses of D-alanine improve affective negative symptoms in patients with schizophrenia (Tsai et al., 2006). Therefore, it is important for future studies to clarify how cognitive effects are modulated by emotional salience and to further characterise changes or reversal of D-alanine effects over time with chronic doses. It is indeed possible that repeated administration with D-alanine may lead to stronger, and clearer effects on cognition.

In addition, the effects of D-alanine were subtle and did not extend to other tasks measuring emotional memory and attention (EMEM and Attentional Dot-Probe) and non-emotional cognitive function (N-Back). No significant effects were seen in the EMEM task, although this is consistent with previous antidepressant drug studies showing that the EREC task has higher sensitivity to treatment with antidepressant drugs (Harmer et al., 2011). Indeed, the lower level of difficulty of the EMEM (recognition task) vs EREC (free recall) may allow for less variability in performance, which would be important to detect more subtle effects.

The lack of findings with the Attentional Dot-Probe, measuring attentional vigilance to emotional vs neutral faces, could be due to high between-subject variability, which is a recognised confound with this task (Schmukle, 2005). The N-Back also showed no significant difference in accuracy or RTs between groups, potentially due to a ceiling effect, with close to perfect accuracy comparing words in the first difficulty level (95\%), and no difference between the second and third levels (75-85\%) (data not shown). Future studies should consider tasks with higher sensitivity to establish a pro-cognitive effect as well as to distinguish between a pure negative bias and a pro-cognitive effect maximised in response to negative cues. For instance, studies could consider tasks measuring executive functioning, such as the Wisconsin Card Sort Task, which has proved sensitive to effects of D-serine in studies with patients with schizophrenia (Tsai et al., 1998).

Contrary to our expectations, D-alanine also had no apparent effect on cortisol. This would suggest that this D-amino acid did not influence emotional processing via the modulation of the hypothalamic-pituitary-adrenal (HPA) axis. However, it is also likely that the variability in salivary cortisol concentrations reduced the sensitivity to detected small but physiologically significant changes. Indeed, the study was performed during the time of day when cortisol naturally declines, and if D-alanine reduced adrenocorticotropic hormone (ACTH) secretion, the concomitant decrease in cortisol may have not been detected when its levels had already reached baseline. This issue might be resolved in future studies by using more sensitive determinations of both ACTH and cortisol in blood as well as saliva, or in investigations of repeated administrations of D-amino acids where the effect of D-alanine on awakening cortisol peaks could be examined (Schmidt et al., 2015).

\section{D-serine did not affect emotional processing or cognition}

The overall performance of individuals on the ETB was not altered by D-serine. The only significant effect observed with D-serine compared to placebo, was the increased accuracy in correctly categorising self-referential words as either 'likeable' or 'dislikeable' in the ECAT. However, the ECAT was not designed to assess cognition or attention per se, as it simply asks participants to categorise words as either likeable or dislikeable. The main outcome measure in this task is the RT to positive vs negative words. It also serves as an encoding task for the subsequent memory recall, which D-serine did not change.

Performance in the Attentional Dot-Probe task was also unaffected by D-serine, which contradicts previous findings by Levin and colleagues (2015) showing that D-serine improved attention/ vigilance. The effect on attention reported by Levin et al. was seen in the Continuous Performance Test-Identical Pairs (CPT-IP), a task where participants have to detect sequential pairs of numbers. In our study, however, we measured attention in response to emotional vs neutral faces. The effects of D-serine in the study by Levin et al. may therefore be specific to a task that measures more pure forms of sustained, non-emotional, attention, which we did not measure to the same extent.

In addition, the current study failed to replicate the enhanced memory effect of D-serine. Levin and colleagues found that D-serine seemed to increase ability to retain information over interference in the Rey Auditory Verbal Learning Test (RAVLT). However, this effect was subtle and mediated by order, 
as performance on D-serine was only superior to placebo when participants received D-serine first. Also, the memory tasks used in the Levin et al. study are not directly comparable with ours; our memory tasks (EREC and EMEM) included words with both a positive and negative valence and not neutral, whereas the task used by Levin et al. (RAVLT) included only neutral words. In addition, in the RAVLT, participants are directly instructed to memorise the words presented, whereas in our tasks there was no such instruction which introduced an element of surprise. Finally, it should be noted that the effects of D-serine in the Levin et al. study did not generalise to all tasks, which emphasises the need for future systematic studies with greater consistency.

Any direct comparisons between our study and previous work is also made difficult by the fact that the Levin et al. study included a cross-over design, a fixed dosage and a higher proportion of males than females. Our dosage was on average higher than that used by Levin and colleagues (range of 2.7-4.9 $\mathrm{g}$ dose vs $2.1 \mathrm{~g}$ fixed dose, respectively), and we included an equal number of males and females. We also decided to use a betweensubjects design rather than cross-over, because of a desire to avoid habituation to the stimuli and maintain task surprise (especially relevant for tasks such as memory recognition and free memory recall). Participants were well matched for baseline characteristics, but we cannot completely exclude the possibility that a cross-over design could have been more sensitive to the effects of D-serine.

The present study also failed to detect any significant differences in subjective report in all the standardised questionnaires used. In the Levin et al. study, D-serine was shown to reduce subjective sadness and anxiety following a single dose. Surprisingly, in our study, in a visual analogue scale administered at the end, participants on D-serine reported feeling less well. However, this analysis included a single item only and there were no baseline measurements for this measure. It is possible that the higher dose used in the current study could have masked any positive effects that may be seen with smaller dosages. Future studies are needed in order to clarify these discrepancies.

\section{Comparing the effects of D-alanine and D-serine}

Given the higher affinity of D-serine for the NMDAR, it is surprising the current study only found significant effects with D-alanine. While this could stem from differences in methodology, it is worth considering these differences in terms of physiological mechanisms. For instance, although an oral dose of D-amino acid elevates its circulating concentration (Kantrowitz et al., 2010; Levin et al., 2015), it is difficult in humans to assess the extent to which this increases synaptic concentrations, and if so, how this effects function. Furthermore, while DAO metabolises both D-serine and $\mathrm{D}$-alanine, it has a greater affinity for the former, and in addition to this, central D-serine is both a product and a substrate for serine racemase which acts bi-directionally and can convert it to L-serine (Balu et al., 2014; Mothet et al., 2015; Wolosker et al., 2008). An alanine racemase has not been detected in mammals and so, conceivably, D-alanine is more stable in the brain, which may explain our findings and why D-alanine was found to have more consistent effects across the battery of tasks used.

Future studies should further examine the effects of D-amino acids in clinical populations. For example, there is evidence that
D-amino acid metabolism is disturbed in mood disorders (Mitani et al., 2006; Zelber-Sagi et al., 2013) and therefore metabolic enzymes could be targeted alongside D-amino acid therapy to increase efficacy (Cho et al., 2016). Plasma D-alanine has been shown to passively increase in patients with schizophrenia on antipsychotics as symptoms improve (Hatano et al., 2010), suggesting D-amino acids might act as biomarkers of a healthy brain. Therefore, D-amino acids might have more effect in subjects with a greater pre-existing metabolic deficit; this might even explain part of the variation in healthy human studies. Comparing the acute effects of D-amino acids in healthy versus diseased participant cohorts would be an interesting future direction.

\section{Limitations}

The current study has limitations worth considering. First, we opted for a between-subject design to avoid habituation to the stimuli and to maintain tasks surprise (especially relevant for the memory tasks). However, it is possible that a cross-over design could have been more sensitive to the effects of D-alanine and $\mathrm{D}$-serine, as this design has the advantage of reducing the influence of confounder variables (because participants serve as their own controls), therefore leading to lower variability in the outcomes. Second, we did not measure plasma levels of circulating D-amino acids. It would have been helpful to determine the participants' baseline levels of both D-alanine and D-serine, to reveal whether pre-existing concentrations confounded the behavioural measures. For instance, high baseline levels may not have been significantly altered by the ingestion of D-amino acids and, therefore, may not have significantly influenced emotional processing and/or cognition. Furthermore, in spite of no effect of D-serine on other glutamate related amino acids (Levin et al., 2015), analysing the full spectrum of circulating amino acids following D-amino acid supplementation would reveal other potential mechanisms e.g. influencing blood tryptophan that would impact on brain serotonin concentrations. Third, the focus of this study was to investigate the psychological effects of D-alanine and D-serine using an acute dosage in healthy volunteers, hence allowing us to assess the psychological mechanisms of these D-amino acids in the absence of any confounding effects of clinical symptoms. However, the clinical relevance of our findings is unknown. Future studies should therefore investigate the effects of both D-alanine and D-serine in clinical populations and using repeated treatment.

\section{Conclusion}

This study is the first to suggest that D-alanine may have a subtle pro-cognitive effect, which seems stronger for negative cues. Further studies will need to dissociate between the presence of a negative bias and/or a cognitive enhancing effect that is maximised in response to negative cues. In contrast with a previous report, we failed to see any significant psychological actions of D-serine. This discrepancy highlights the importance of considering the use of similar, standard tasks across studies. There is also need for further insight into the synaptic metabolism of NMDA co-agonists and their mechanism in humans to develop effective therapies. Future studies could consider chronic D-amino acid regimes and tasks that more precisely isolate cognitive effects, combined with imaging techniques such as functional Magnetic 
Resonance Imaging (fMRI) to detect changes independent of behaviour.

\section{Acknowledgements}

The authors would like to thank all of the volunteers who participated in the study. They would also like to thank Clare Williams for preparing the drinks to be administered to participants, and Ann Sharpley for help with randomisation.

\section{Declaration of conflicting interests}

The authors declared the following potential conflicts of interest with respect to the research, authorship and/or publication of this article: LPC and $\mathrm{CJH}$ are supported by the NIHR Oxford Health Biomedical Research Centre, UK. CJH reives consultancy fees from p1vital, Lundbeck, Sage Therapeutics and J\&J. All remaining authors have no conflicts to disclose.

\section{Funding}

The authors disclosed receipt of the following financial support for the research, authorship and/or publication of this article: This work was funded by internal University of Oxford funds (PWJB). The views expressed are those of the authors and not necessarily those of the National Health Service (NHS), the National Institute of Health Research (NIHR) or the Department of Health.

\section{ORCID iDs}

Mia A Thomaidou (iD https://orcid.org/0000-0002-2237-9459

Philip WJ Burnet iD https://orcid.org/0000-0002-6374-8054

\section{Supplemental material}

Supplemental material for this article is available online.

\section{References}

Arnone D, Horder J, Cowen PJ, et al. (2009) Early effects of mirtazapine on emotional processing. Psychopharmacology (Berl) 203: 685-691.

Balu DT, Takagi S, Puhl MD, et al. (2014) D-serine and serine racemase are localized to neurons in the adult mouse and human forebrain. Cell Mol Neurobiol 34: 419-435.

Bennett A O MR (2008) Stress and anxiety in schizophrenia and depression: Glucocorticoids, corticotropin-releasing hormone and synapse regression. Aust N Z J Psychiatry 42: 995-1002.

Berman RM, Cappiello A, Anand A, et al. (2000) Antidepressant effects of ketamine in depressed patients. Biol Psychiatry 47: 351-354.

Browning M, Reid C, Cowen PJ, et al. (2007) A single dose of citalopram increases fear recognition in healthy subjects. $J$ Psychopharmacol 21: 684-690.

Capitão LP, Murphy SE, Browning M, et al. (2015) Acute fluoxetine modulates emotional processing in young adult volunteers. Psychol Med 45: 2295-2308.

Chan SY, Matthews E and Burnet PWJ (2016) ON or OFF? Modulating the N-methyl-D-aspartate receptor in major depression. Front Mol Neurosci 9: 169.

Cho S-E, Na K-S, Cho S-J, et al. (2016) Low d-serine levels in schizophrenia: A systematic review and meta-analysis. Neurosci Lett 634: $42-51$.

Coyle JT (2012) NMDA receptor and schizophrenia: A brief history. Schizophr Bull 38: 920-926.

First MB, Spitzer RL, Gibbon M, et al. (1996) Structured Clinical InterView for DSM-IV Axis I Disorders, Clinician Version (SCID-CV). Washington, DC: American Psychiatric Press.
Girshkin L, Matheson SL, Shepherd AM, et al. (2014) Morning cortisol levels in schizophrenia and bipolar disorder: A meta-analysis. Psychoneuroendocrinology 49: 187-206.

Grunze HC, Rainnie DG, Hasselmo ME, et al. (1996) NMDA-dependent modulation of CA1 local circuit inhibition. JNeurosci 16: 2034-2043.

Guercio GD and Panizzutti R (2018) Potential and challenges for the clinical use of d-serine as a cognitive enhancer. Front Psychiatry 9: 14.

Hamase K, Konno R, Morikawa A, et al. (2005) Sensitive determination of D-amino acids in mammals and the effect of D-amino-acid oxidase activity on their amounts. Biol Pharm Bull 28: 1578-1584.

Harmer CJ, Bhagwagar Z, Perrett DI, et al. (2003) Acute SSRI administration affects the processing of social cues in healthy volunteers. Neuropsychopharmacology 28: 148-152.

Harmer CJ, Cowen PJ and Goodwin GM (2011) Efficacy markers in depression. J Psychopharmacol 25: 1148-1158.

Harmer CJ, Shelley NC, Cowen PJ, et al. (2004) Increased positive versus negative affective perception and memory in healthy volunteers following selective serotonin and norepinephrine reuptake inhibition. Am J Psychiatry 161: 1256-1263.

Hashimoto K, Malchow B, Falkai P, et al. (2013) Glutamate modulators as potential therapeutic drugs in schizophrenia and affective disorders. Eur Arch Psychiatry Clin Neurosci 263: 367-377.

Hatano T, Ohnuma T, Sakai Y, et al. (2010) Plasma alanine levels increase in patients with schizophrenia as their clinical symptoms improve-Results from the Juntendo University Schizophrenia Projects (JUSP). Psychiatry Res 177: 27-31.

Herbert J (2013) Cortisol and depression: Three questions for psychiatry. Psychol Med 43: 449-469.

Javitt DC (2009) When doors of perception close: Bottom-up models of disrupted cognition in schizophrenia. Annu Rev Clin Psychol 5: 249-275.

Kantrowitz JT, Malhotra AK, Cornblatt B, et al. (2010) High dose D-serine in the treatment of schizophrenia. Schizophr Res 121: 125-130.

Labrie V, Clapcote SJ and Roder JC (2009a) Mutant mice with reduced NMDA-NR1 glycine affinity or lack of D-amino acid oxidase function exhibit altered anxiety-like behaviors. Pharmacol Biochem Behav 91: 610-620.

Labrie V, Duffy S, Wang W, et al. (2009b) Genetic inactivation of D-amino acid oxidase enhances extinction and reversal learning in mice. Learn Mem 16: 28-37.

Levin R, Dor-Abarbanel AE, Edelman S, et al. (2015) Behavioral and cognitive effects of the N-methyl-D-aspartate receptor co-agonist D-serine in healthy humans: Initial findings. $J$ Psychiatr Res 61: 188-195.

Lohmann C and Kessels HW (2014) The developmental stages of synaptic plasticity. J Physiol 592: 13-31.

MacKay M-AB, Kravtsenyuk M, Thomas R, et al. (2019) D-Serine: Potential therapeutic agent and/or biomarker in schizophrenia and depression? Front Psychiatry 10: 25.

Malkesman O, Austin DR, Tragon T, et al. (2012) Acute D-serine treatment produces antidepressant-like effects in rodents. Int J Neuropsychopharmacol 15: 1135-1148.

Meuwese JDI, van Loon AM, Scholte HS, et al. (2013) NMDA receptor antagonist ketamine impairs feature integration in visual perception. PloS One 8: e79326.

Mitani H, Shirayama Y, Yamada T, et al. (2006) Correlation between plasma levels of glutamate, alanine and serine with severity of depression. Prog Neuropsychopharmacol Biol Psychiatry 30: 11551158.

Miyoshi Y, Hamase K, Okamura T, et al. (2011) Simultaneous twodimensional HPLC determination of free D-serine and D-alanine in the brain and periphery of mutant rats lacking D-amino-acid oxidase. J Chromatogr B Analyt Technol Biomed Life Sci 879: 3184-3189. 
Mothet J-P, Le Bail M and Billard J-M (2015) Time and space profiling of NMDA receptor co-agonist functions. J Neurochem 135: 210-225.

Noordewier MK and Breugelmans SM (2013) On the valence of surprise. Cogn Emot 27: 1326-1334.

Rubio MD, Drummond JB and Meador-Woodruff JH (2012) Glutamate receptor abnormalities in schizophrenia: Implications for innovative treatments. Biomol Ther (Seoul) 20: 1-18.

Sanacora G, Zarate CA, Krystal JH, et al. (2008) Targeting the glutamatergic system to develop novel, improved therapeutics for mood disorders. Nat Rev Drug Discov 7: 426-437.

Schmidt K, Cowen PJ, Harmer CJ, et al. (2015) Prebiotic intake reduces the waking cortisol response and alters emotional bias in healthy volunteers. Psychopharmacology (Berl) 232: 1793-1801.

Schmukle SC (2005) Unreliability of the dot probe task. Eur J Personal 19: 595-605.
Tsai G, Yang P, Chung LC, et al. (1998) D-serine added to antipsychotics for the treatment of schizophrenia. Biol Psychiatry 44: 10811089.

Tsai GE, Yang P, Chang Y-C, et al. (2006) D-alanine added to antipsychotics for the treatment of schizophrenia. Biol Psychiatry 59: 230-234.

Winder-Rhodes SE, Chamberlain SR, Idris MI, et al. (2010) Effects of modafinil and prazosin on cognitive and physiological functions in healthy volunteers. J Psychopharmacol 24: 1649-1657.

Wolosker H, Dumin E, Balan L, et al. (2008) D-amino acids in the brain: D-serine in neurotransmission and neurodegeneration. FEBS J 275 : 3514-3526.

Zelber-Sagi S, Toker S, Armon G, et al. (2013) Elevated alanine aminotransferase independently predicts new onset of depression in employees undergoing health screening examinations. Psychol Med 43: 2603-2613. 